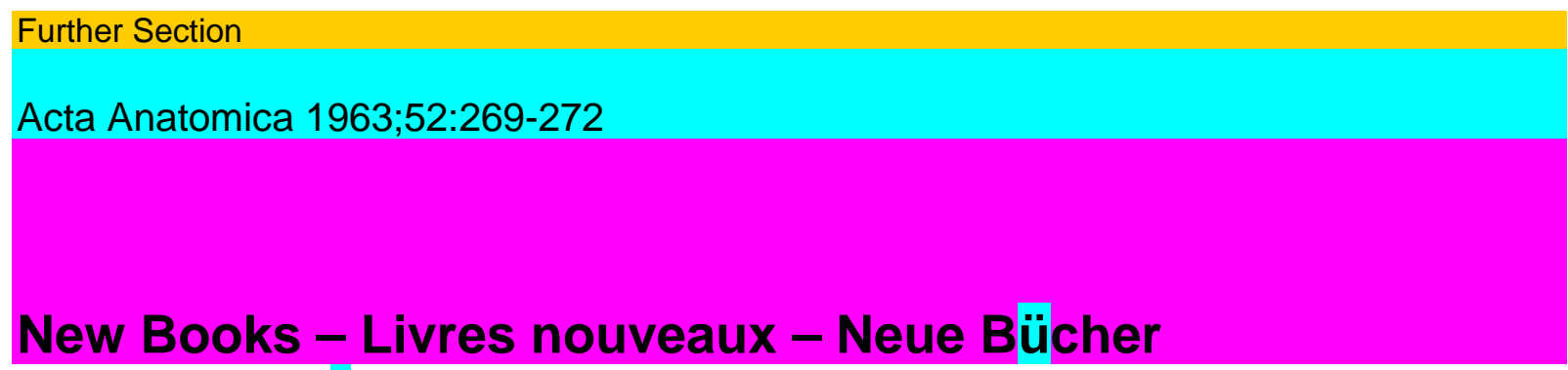

H. Barthel: Mißbildungen des menschlichen Herzens. Entwicklungsgeschichte und Pathologic Thieme, Stuttgart 1960. XII + 237 S., 215 z.T. farb. Abb. Ln.

DM 188.--

Um gleich den wichtigsten Eindruck vorwegzunehmen: Barthel hat mit seiner Darstellung der Entwicklungsgeschichte und der Mißbildungen des menschlichen Herzens ein außergewöhnlich interessantes, inhaltsreiches, zum Nachdenken und manchmal auch zu kritischen Überlegungen anregendes Werk geschrieben. Und dies bei weitem nicht nur für interne und chirurgische Herzspezialisten. Auch Anatomen und Embryologen werden darin eine Fülle von Hinweisen finden, die ihr persönliches Wissen und die Gestaltung des Unterrichts bereichern. Der knappe, klare, prägnant geschriebene Text liest sich wie eine Legende zu den zeichnerisch und didaktisch hervorragenden, in bezug auf Anzahl, Format und Druck äußerst großzügig ge-stalteten Abbildungen. Autor, Zeichnerin und Verlag haben hier wirklich keine Mühe und keinen Aufwand gescheut, um das infolge der komplizierten morphologischen Verhältnisse so anspruchsvolle Thema anschaulich - und manchmal geradezu genuß-voll - verabreichen zu können.

Die Darstellung der Herzentwicklung ist für Lehrer und Schüler bekanntlich oft eine harte Nuß. Der Tendenz zur Vereinfachung und Schematisierung steht heute die Notwendigkeit gegenüber, den Medizinern die Grundlagen für das Verständnis der vielfältigen, praktisch bedeutsamen Mißbildungen in logischem Zusammenhang zu vermitteln. Ohne Zweifel haben gerade in dieser Beziehung die auf die hämodyna-mischen Verhältnisse ausgerichteten Modellversuche manche Erleichterung ge-bracht. Sie stehen denn auch im Brennpunkt der von Barthel vertretenen Anschau-ungen. Seine eigenen, mit imponierend einfachen Mitteln ausgeführten Modellversuche haben etwas Bestechendes an sich und bilden eine wertvolle Ergänzung, teil-weise auch eine Berichtigung der von Kl.Goerttler an Glasmodellen embryonaler Herzanlagen erhobenen Befunde. So ziehen sich - überspitzt formuliert - ein roter und ein blauer Faden durch das ganze, in seinen Einzelprozessen oft so verwirrende und komplexe Geschehen: Die das linke und das rechte Herz schon in frühen Ent-wicklungsstadien prägende Strombahn. Trotz seiner persönlichen, an vielen Stellen deutlich spürbaren Begeisterung für diese Modellversuche bemüht sich der Autor aber, auch die grundlegenden Arbeiten von Spítzer, Pernkopf und Wírtinger, Shaner und vieler anderer in das Gesamtbild einzuordnen, soweit dies ohne Widersprüche möglich ist. So fühlt sich jeder, der das Buch aufmerksam gelesen hat, dem Verständnis der Herzentwicklung näher als je zuvor, auch wenn er sich vielleicht man-chen Schlußfolgerungen und Hypothesen gegenüber eine gewisse kritische Zurück-haltung auferlegt. Eine Buchbesprechung ist aber nicht der Ort, um diese theoreti-schen Aspekte zu erörtern. Bei der Aktualität des Themas dürften sich berufene Fachgelehrte über kurz oder lang mit einigen Punkten auseinandersetzen. Eine grundsätzliche Überlegung sei hingegen nicht verschwiegen: In der ganzen Anlage erinnert die Betrachtungsweise an die Arbeiten von Pauwels über die Differenzierung der Stützgewebe, was von Barthel übrigens gebührend erwähnt wird. Gerade dieser Vergleich deckt aber eine schwache Stelle auf: Das was den theoretischen Über- 


\section{New Books - Livres nouveaux - Neue Bücher}

legungen von Pauwels ihr Gewicht gibt, sind die immer wieder zwischen die Modell-versuche eingestreuten histologischen, experimentell morphologischen und klini-schen Dokumente. Und an dieser Stelle hat der Autor - gemessen an der großzügi-gen Illustration des Buches - gespart. Didaktisch und künstlerisch hat die nur aus-nahmsweise auf Einzelfälle bezugnehmende Illustrationstechnik bestimmt ihre Vor-teile. An Uberzeugungskraft hätte dagegen manches Argument gewonnen, wenn zwischen Modellaufnahme und idealisierter zeichnerischer Darstellung das anato-mische oder histologische Präparat Platz gefunden hätte. An Möglichkeiten dazu fehlt es nicht, so beispielsweise bei der Primitiventwicklung des Herzens (das von Kl.Goerttler übernommene Schema in Abb. 1 ist sowohl für das Hühnchen wie für den Menschen nicht zutreffend), bei der für die Hypothese über die Septierung und Klappenentwicklung so bedeutsamen Gallertschicht, bei der Faserdifferenzierung innerhalb der Endokardkissen, der Entwicklung der Lungenvenen - um nur einige Teilprozesse anzudeuten. In ähnlichem Sinne ist auch der Wunsch nach einer besse-ren Verankerung der Literaturangaben im Text und in den Bildunterschriften zu verstehen, namentlich auch bei den einzelnen Mißbildungsfällen. In dieser Hinsicht erfüllt das Werk vielleicht nicht ganz alle Ansprüche, welche der Grundlagenfor-scher - und an diesen wendet sich ja die Rezension in einer anatomischen Fachzeit-schrift - an eine derartige Monographie stellen darf. Doch tut dies unserer eingangs formulierten Gesamtbeurteilung keinerlei Abbruch, und wir sind überzeugt, daß jeder, der sich mit der normalen und gestörten Entwicklung des menschlichen Herzens beschäftigt, das prächtige Werk mit großem Gewinn zu Rate ziehen wird.

Robert Schenk (Basel)

Medizinische Grundlagenforschung, Band II. Hg. v. K. Fr. Bauer. Thieme, Stuttgart 1959. VIII + 827 p., 187 fig., 63 tab., Ln. DM 168.-.

Der Referent hat bereits bei Besprechung von Band I der «Medizinischen

Grundlagenforschung», damals noch «Ergebnisse der Medizinischen Grundlagenforschung», sich eindeutig zu diesem Werk bekannt und die Ansicht vertreten, daß bei dem raschen Erkenntnisfortschritt unserer Tage Sammelwerke wie das vorlie-gende dazu berufen seien, das klassische, leider nur allzu rasch veraltende «Hand-buch» vollwertig zu ersetzen. Das Prinzip, hervorragende Wissenschaftler auf ihrem Spezialgebiet zu Wort kommen zu lassen, ist auch im vorliegenden Band realisiert. Nennen wir unter den 17 Beiträgen nur diejenigen, die das besondere Interesse des Morphologen beanspruchen. Es sind dies die Ausführungen von R.Granít «Über die absteigenden Einflüsse der Formatio reticularis, insbesondere in bezug auf die Gamma-Neuronen», diejenigen von H.-Ch.Lüttgau über «Die Physiologie der markhaltigen Nervenfaser» von E. C. Crosby, T. Humphrey und M. J. Showers über « Einige Anordnungen, Verbindungen und Funktionen der supplementären motorischen Rinden», von E. Hagen «Über morphologische Veränderungen vegetativer Nervenzentren bei pathologischen Vorgängen», von K.Fr. Bauer und E.Müller über «Die Zellenlehre», von G. Ruhenstroth-Bauer und P.Sachtleben über «Die Zellelektrophorese» und schließlich von R. Haase über « Strukturbildung in der Natur und der Zweite Hauptsatz der Thermodynamik». Ein ausgezeichnetes Sachregister beschließt den gehalt- und wertvollen Band, der trotz seinem nicht geringen Preis weiteste Verbreitung zu finden verdient und zweifellos auch finden wird. G. Wolf-Heidegger (Basel) New Books - Livres nouveaux - Neue Bücher 271 Biological Structure and Function, Vol. 1 and Vol. 2. Ed. by W. T. Goodwin and 
O.Lindberg. Academic Press, London 1961. Vol. 1: XII+ 363 p., ill., 70 sh.

Vol.2: XVI+ 665 p., ill., 126 sh.

Goodwin und Lindberg haben sich der sehr verdienstvollen Aufgabe unterzogen, die

Verhandlungen des 1. von der International Union of Biochemistry und der International Union of Biological Science im September 1960 in Stockholm abge-haltenen 1. Symposiums über «Biological Structure and Function* - und zwar die Vorträge mit den Diskussionsbemerkungen - in 2 gehaltvollen, umfangreichen Bän-den herauszugeben.

Der 1. Band beginnt mit einer Einleitung von A. Tíselíus und enthält eine größere Zahl von zum Teil sehr wichtigen Beiträgen zu den Themen: Makromole-kularstruktur und Funktion, Mikrosomen und Proteinsynthese sowie Polysaccha-ride. Dabei sei hervorgehoben, daß nicht nur in den Beiträgen selbst, sondern zum Teil auch in den Diskussionsbemerkungen wichtige, für den Spezialisten bedeutsame Feststellungen und Anregungen enthalten sind.

Während die Vorträge des 1. und 3. Abschnittes vorwiegend den Biochemiker interessieren, findet der Morphologe im Kapitel «Mikrosomen und Proteinsynthese » eine Reihe wichtiger Beiträge; erwähnen wir aus der großen Zahl nur die beiden ein-leitenden Vorträge von K.R.

Porter über: «The Endoplasmic Reticulum: Some Current Interpretations of its Form and Functions* und von H.Holter über «Pino-cytosis».

Die Kapitel von Band 2 befassen sich mit der «Mitochondrial Structure and Function», der «Structure and Function of Chloroplasts and Chromatophores» der «Intact Cellular Structure and Function* und dem «Specific Membrane Transport and its Adaptation*. Die Fülle der zum großen Teil hochinteressanten Beiträge macht es dem Referenten unmöglich, auf Einzelheiten einzugehen, genügen doch bereits die Titel der verschiedenen Abschnitte - von der großen Anzahl der Beiträge und den zum Teil klangvollen Namen der Autoren ganz abgesehen - um darzutun, daß die beiden Bände in die Bibliothek jedes ultrastrukturell und biochemisch interessierten - also jedes modernen - Morphologen gehören. Druck und Ausstattung beider Bände sind vorzüglich. Der Preis ist in Anbetracht der heutigen Publikatíonskosten nicht als übersetzt zu bezeichnen. G. Wolf-Heidegger (Basel) Recent Advances in Anatomy, Second Series. Ed. by F. Goldby and R. J. Harrison. Churchill, London 1961. VI+ 477 p., ill., 60 sh.

Während es der Generation unserer Vorgänger und insbesondere ihren Lehrern noch möglich war, den Überblick über alle Teilgebiete unseres Faches zu gewinnen und vor allem auch zu behalten, führt der rasante Erkenntnisfortschritt unweiger-lich zur Spezialisierung. Dabei wird es selbst für den Spezialisten heute zunehmend schwieriger, aus der Literaturfülle das wirklich Bedeutsame herauszulesen, ganz besonders aber noch das zu erfassen, was außerhalb seines engeren Interessens- und Arbeitsgebietes an wichtigen neuen Erkenntnissen veröffentlicht wird. Es ist des-halb besonders verdienstvoll, daß die beiden Londoner Anatomen F.Goldby und R.J.Harrison, unterstützt von einem Team ausgezeichneter Fachleute sich der Aufgabe unterzogen haben, 34 Jahre nachdem Woollard den 1. Band der «Recent Advances in Anatomy* herausgegeben hat, nun einen 2. Band der Serie folgen zu lassen.

Ein Vielmännerbuch bringt es mit sich, daß im allgemeinen nicht alle Kapitel hinsichtlich didaktischem Geschick und Inhalt gleichwertig sein können. Kami man

272 New Books - Livres nouveaux - Neue Bücher daher dem Buch ein besseres Lob zollen, als zu sagen, daß die Lektüre jedes der 14 Abschnitte dem Leser reichen Gewinn und insbesondere mannigfache, wichtige Anregung zu weiterem 
Studium der entsprechenden Originalliteratur vermittelt. Nennen wir im folgenden Titel und Verfasser der verschiedenen Abschnitte:

The cell and the cytology of exocrine secretion (C.L.Foster); Endocrine glands: adrenal, thyroid, pancreas, chromaffin tissue (J.D. Lever); anterior pituitary (R. J. Harrison); Cells in reproduction and reproductive organs (R.J. Harrison); Some aspects of mammalian embryology

(R.J.Harrison); Epithelia: characteristics and repair (F. R. Johnson); Bone and bones (J. J. Pritchard); The fine structure of enamel (R.W. Fearnhead); The nerve cell (F.Goldby); The Schwann cell, myelin and other tissues associated with nerve cells (_F. Goldby); Nerve endings (F. Goldby); Some recent work on the brain and spinal cord (F. Goldby); Liver, spleen and kidney with special reference to the blood supply (C.H. Rarnett and O.J.Lewis); Joints and movement (C.H. Barnett); Broncho-pulmonary segments and the alveolar lining (R. J. Harrison); The evolution of the higher primates (J. R. Napier).

Es ist erstaunlich, daß es den Autoren da, wo es angezeigt erschien, gelungen ist, in dem gebotenen kurzen Rahmen sogar einen Einblick in die Fülle der Ul·trastrukturforschung unserer Tage zu bieten. Die mehr oder weniger umfangreichen Literaturverzeichnisse am Schluß der einzelnen Abschnitte sowie ein vielleicht etwas kurzes, aber durch seine geschickte Zusammenstellung doch recht brauchbares Sachregister erhöhen den Wert des Buches, das für jeden Morphologen vor allem zur Vervollständigung seines Fachwissens für Unterrichtszwecke ein unentbehrliches Kompendium darstellt und darüber hinaus wertvolle Anregungen vermittelt. Der Preis des gut ausgestatteten Buches darf in Anbetracht der heutigen Publikationskosten als durchaus angemessen bezeichnet werden. G. Wolf-Heidegger (Basel) 